\title{
SKA2 promotes proliferation and invasion of hepatocellular carcinoma cells via activating the $\beta$-catenin signaling pathway
}

\author{
D. WANG ${ }^{1}$, Y. J. SUO 2 , L. GONG 3 , S. Q. $\mathrm{LV}^{1, *}$ \\ ${ }^{1}$ VIP ward, Affiliated Hospital of Traditional Chinese Medicine, Xinjiang Medical University, Urumqi, China; ${ }^{2}$ Department of Critical Care \\ Medicine, Affiliated Hospital of Traditional Chinese Medicine, Xinjiang Medical University, Urumqi, China; ${ }^{3}$ Department of Geriatrics, Affili- \\ ated Hospital of Traditional Chinese Medicine, Xinjiang Medical University, Urumqi, China
}

${ }^{*}$ Correspondence: lvshuqin83@163.com

Received July 9, 2019 / Accepted September 18, 2019

\begin{abstract}
Spindle and kinetochore associated complex subunit 2 (SKA2) is a part of the spindle and kinetochore associated (SKA) complex, which has been reported in various cancers, including the breast cancer, lung cancer, and glioma. However, its role remains unclear in hepatocellular carcinoma (HCC). Our study found that SKA2 mRNA levels and immunohistochemistry staining were significantly increased in HCC tissues compared with normal tissues. The role of SKA2 in cell proliferation and invasion was also determined. Overexpression of SKA2 significantly promoted cell proliferation and invasion, while knocking down of SKA2 expression inhibited the growth and invasion of HCC cells. In experiments investigating the underlying mechanism, overexpression of SKA2 may increase the expression levels of total $\beta$-catenin, and knockdown of SKA2 may decrease the expression levels of total $\beta$-catenin. Our studies thus suggest that SKA2 may promote proliferation and invasion of hepatocellular carcinoma cells by activating the $\beta$-catenin signaling pathway, which can serve as a potential target in the diagnosis and/or treatment of HCC.
\end{abstract}

Key words: SKA2, proliferation, invasion, hepatocellular carcinoma, $\beta$-catenin

Hepatocellular carcinoma (HCC) is one of the most common malignant tumors with high morbidity and mortality. It is the fifth most commonly occurring cancer and the third leading cause of cancer deaths worldwide [1]. Multiple treatments such as liver resection, transarterial chemoembolization (TACE), radiotherapy and sorafenib can be employed for HCC patients. However, the prognosis of HCC patients remains poor due to the prognosis difficulty, high recurrence and early vascular invasion $[2,3]$. More and more evidence revealed that HCC was associated with multigene mutation [4], therefore, the molecular target is now as a novel therapy for advanced HCC patients, as it showed a favorable curative effect and significantly prolonged the patient's survival time [5]. Hence, understanding HCC development and finding new molecular targets for HCC treatment become a significant focus.

Spindle and kinetochore associated complex subunit 2 (SKA2) located on human chromosome $17 \mathrm{q} 23.2$ is a part of the spindle and kinetochore associated (SKA) complex, a heterotrimeric complex formed from SKA1, SKA2, and SKA3 $[6,7]$. SKA2 is involved in the formation of Ska complex and plays an important role in the maintenance of the mitotic mid-plateau and shutdown of the spindle checkpoint [6, $8-10]$. Recent evidence showed that overexpression of SKA2 promoted the proliferation of human breast cancer, whereas SKA2 knockdown in human lung epithelial cells reduced transactivation and suppressed dexamethasone inhibition of proliferation $[11,12]$. Furthermore, the function of SKA2 has been observed in various cancers, including breast cancer, lung cancer, and glioma [13-16]. However, the role of SKA2 in HCC still remains unclear.

In our study, we focused on the importance of SKA2 in the progression and metastasis of HCC. We demonstrated that SKA2 was highly expressed in human HCC tissues and enhanced the proliferation and invasion of HCC cell lines in vitro. Additionally, we evaluated the molecular mechanism of SKA2 in HCC growth and metastasis.

\section{Patients and methods}

HCC tissue samples. Thirty-five pairs of primary HCC tissues and their paired adjacent normal tissues were obtained from patients who underwent hepatectomy between February 2017 and July 2018 at the Affiliated 
Hospital of Traditional Chinese Medicine, Xinjiang Medical University. All of them signed informed consent. All specimens were immediately snap-frozen in liquid nitrogen and maintained at $-80^{\circ} \mathrm{C}$ until use. These 30 paired samples were subjected to RNA extraction for quantitative real time PCR (qRT-PCR) analysis, and the other 5 paired samples were used for immunohistochemistry. Ethical approval for the study was obtained from the Research Ethics Committee of the Affiliated Hospital of Traditional Chinese Medicine, Xinjiang Medical University.

Immunohistochemistry. Clinical HCC tissues and paired non-cancerous tissues were fixed in formalin, embedded in paraffin, and cut into $5 \mu \mathrm{m}$ thick consecutive sections. After deparaffinization and antigen recovery (in a sodium citrate solution, $\mathrm{pH} 6.0,20 \mathrm{~min}, 98^{\circ} \mathrm{C}$ ), the sections were washed thrice with $0.01 \mathrm{~mol} / \mathrm{l} \mathrm{PBS}$ for $5 \mathrm{~min}$ each time, blocked for $1 \mathrm{~h}$ in $0.01 \mathrm{~mol} / \mathrm{l} \mathrm{PBS}$ containing $0.3 \%$ Triton X-100 and 5\% BSA, and incubated with an anti-SKA2 (1:100, ab75345, Abcam) antibody at $4{ }^{\circ} \mathrm{C}$ overnight. After brief washes with $0.01 \mathrm{~mol} / \mathrm{l} \mathrm{PBS}$, the sections were incubated with $0.01 \mathrm{~mol} / \mathrm{l}$ PBS containing a horseradish peroxidase-conjugated antirabbit IgG antibody (1:500, SA00001-2, Proteintech) for 2 $h$, followed by development with $0.003 \% \mathrm{H}_{2} \mathrm{O}_{2}$ and $0.03 \% 3$, 30-diaminobenzidine in $0.05 \mathrm{~mol} / \mathrm{l}$ Tris- $\mathrm{HCl}(\mathrm{pH} 7.6)$. Two histopathologists blindly reviewed the slides and scored the staining.

RNA preparation and real-time PCR. Preparation of RNA and complementary DNA (cDNA) samples were performed as described earlier [17]. The specimens were snap-frozen in liquid nitrogen and stored at $-80^{\circ} \mathrm{C}$. The total RNA of tumor tissues and adjacent noncancerous tissues from the 30 patients was isolated using Trizol reagent (Invitrogen, Carlsbad, CA), and reverse-transcribed to cDNA using the PrimeScript RT reagent Kit (DRR037A, Takara, Japan) according to the manufacturer's instructions. The real-time PCR was performed using the SYBR Premix Ex Taq (Takara). The SKA2-specific primers were 5'-GGAACTGATGTTCCAGAAAGCTG-3' (forward) and 5'-AGCTCCAGGTCTGTTTGCTT-3' (reverse), and the GAPDH primers were 5'-ATGACCCCTTCATTGACCTCA-3' (forward) and 5'-GAGATGATCACCCTTTTGGCT-3'(reverse). GAPDH acted as an internal control. The relative mRNA level of target genes to that of GAPDH in clinical samples was calculated according to the methods described [18].

Cell culture. Human HCC cell lines YY-8103, YY-8103, HepG2, and Huh7 were purchased from the cell bank of the Shanghai Biology Institute, Chinese Academy of Science (Shanghai, China). Cell lines were maintained in a humidified atmosphere containing $5 \% \mathrm{CO}_{2}$ at $37^{\circ} \mathrm{C}$ in $\mathrm{DMEM}$ medium supplemented with $100 \mathrm{U} / \mathrm{ml}$ penicillin, $100 \mathrm{mg} / \mathrm{ml}$ streptomycin, and 10\% fetal bovine serum (FBS; Gibco).

Cell transfection. Lentiviral constructs of SKA2 and the nucleotide sequences of shRNA against SKA2 were described previously [19]. The sequences of the shSKA2 were 5'-CCGGCAAACTTTGTATGCCCGCTTTCTCGAGA-
AAGCGGGCATACAAAGTTTGTTTTTG-3' (forward) and 5'-AATTCAAAAACAAACTTTGTATGCCCGCTTTCTCGAGAAAGCGGGCATACAAAGTTTG-3' (reverse). YY-8103 and LM3 cells were infected with p23-ZsGreenSKA2 and HepG2 and Huh7 cells were infected with pLKO.1-shRNA. Overexpressed and silenced cells were sorted using flow cytometry or selected by puromycin $(4 \mu \mathrm{g} /$ $\mathrm{ml}$ ) for at least 4 days. All cells were routinely cultured in DMEM (Invitrogen) supplemented with $10 \%$ fetal bovine serum (FBS; Gibco) in a humidified incubator containing 5\% $\mathrm{CO}_{2}$ at $37^{\circ} \mathrm{C}$.

Western blot. Western blot analysis was performed as previously described [20]. Total cell lines protein was harvested and lysed with RIPA buffer (Beyotime, Beijing, China) according to the manufacturers' instructions. Primary antibodies against the following proteins were used as follows: SKA2 from Abcam; $\beta$-catenin (cat. \#8480), Flag (cat. \#14793) and GAPDH (cat. \#5174) were purchased from Cell Signaling Technology. The images were captured using the Gel Dox XR system (Bio-Rad, Philadelphia, PA).

Cell proliferation analysis. Cell proliferation was detected with crystal violet assay or MTT staining method described by manufacturers' instructions. Briefly, in crystal violet assay, $1 \times 10^{3}$ cells were seeded into 6 -well plates, the cells were cultured in medium with $10 \%$ FBS, changed the medium every three days and 2 weeks later the cells were stained with crystal violet. After staining for $10 \mathrm{~min}$, the fixed cells were washed with phosphate-buffered saline (PBS) and photographed. Then, $1 \mathrm{ml}$ glacial acetic acid was added to the cells and the optical density (OD) was detected at $570 \mathrm{~nm}$ by a microplate reader.

In MTT assay, $1 \times 10^{3}$ cells were seeded into 96-well plates, and cell viability was detected by MTT, after $1,2,3,4,5,6$, or 7 -day incubation, $20 \mu \mathrm{l}$ of a $5 \mathrm{mg} / \mathrm{ml}$ MTT solution was added to each well, and the plate was incubated at $37^{\circ} \mathrm{C}$ for further 4 hours. Then, the medium was removed, $200 \mu \mathrm{l}$ DMSO was added to dissolve the generated formazan. The measurement process was performed every 24 hours for 7 days to generate a cell growth curve. The optical density (OD) was measured at $490 \mathrm{~nm}$ by a microplate reader.

Transwell assay. The invasion of HCC cells was examined by transwell with polyethylene terephthalate membranes (24-well inserts, $8.0 \mu \mathrm{m}$, Corning). $150 \mu \mathrm{l}$ cell suspension contained $2 \times 10^{5}$ cells were loaded into the upper well coated with Matrigel (BD Biosciences). Next, $500 \mu \mathrm{l}$ DMEM medium with $10 \%$ FBS was placed into the bottom of the well to induce invasion. $72 \mathrm{~h}$ later, invasive cells were stained with $0.1 \%$ crystal violet. Randomly selected four fields and counted the cells, experiments were repeated three times and the data are presented as the means \pm SD.

Statistical analysis. Statistical evaluations were performed with GraphPad software (www.graphpad.com), and results were shown as mean \pm SD unless otherwise stated. Statistical significance was set at a $\mathrm{p}$ value of $<0.05$ and marked with an asterisk. 


\section{Results}

SKA2 is upregulated in HCC tissues. Firstly, we examined the mRNA level of SKA2 in 30 pairs of HCC tissues and the corresponding normal tissue, and we found that the mRNA expression of SKA2 was significantly increased in 22 of 30 HCC tissues compared to the matched normal tissues (Figure 1A). The immunohistochemistry assays showed that SKA2 was mainly located in the cytoplasm, and the tumor tissues showed stronger staining when compared to the paired normal tissues in five patient cases (Figures 1B, S1), which was consistent with the results of real-time PCR. From the TCGA database, we found that the expression of SKA2 was significantly upregulated in 11\% (40/360) HCC tissues (Figure 1C). In addition, the overall survival (OS) rate was significantly higher in cases without SKA2 alteration group compared to cases with SKA2 alteration group ( $<<0.05$, Figure 1D). Taken together, we can confirm that SKA2 is upregulated in HCC tissues based on our analysis and TCGA database.

Successful overexpression and knockdown of SKA2 in HCC cell lines. Based on the clinical data, we hypothesized that SKA2 might influence the growth of HCC cells. Firstly, we checked the SKA2 levels in several common HCC cell lines at the protein level. We found that the expression level of SKA2 was significantly increased in HepG2 and Huh7 cell lines compared to YY-8103 and LM3 cell lines (Figure 2A). To identify the function of SKA2 in HCC cells, we transfected the plasmids containing either an empty p23 vector or SKA2 overexpression vectors (Flag-SKA2) into YY-8103 and LM3 HCC cell lines, which have relatively low expression of SKA2. And we used specific shRNA targeting SKA2 in HepG2 and Huh7 cell lines, which have relatively high expression of SKA2. Western blotting analyses showed that we successfully established the overexpression and knockdown of SKA2 in HCC cell lines (Figures 2B-E).

SKA2 overexpression promoted growth and invasion of HCC cell lines. The MTT assays showed that the absorbance values of the LM3 cells after 4, 5 and 6 days post-transfection with SKA2 overexpression vectors was significantly higher than those of the untreated cells $(p<0.01$, Figure $3 A)$. Similarly, the absorbance values of the YY- 8103 cells after 5 and 6 days post-transfection with SKA2 overexpression vectors were also significantly higher than those of the untreated cells $(\mathrm{p}<0.01$, Figure $3 \mathrm{~A})$. Furthermore, the crystal violet assays

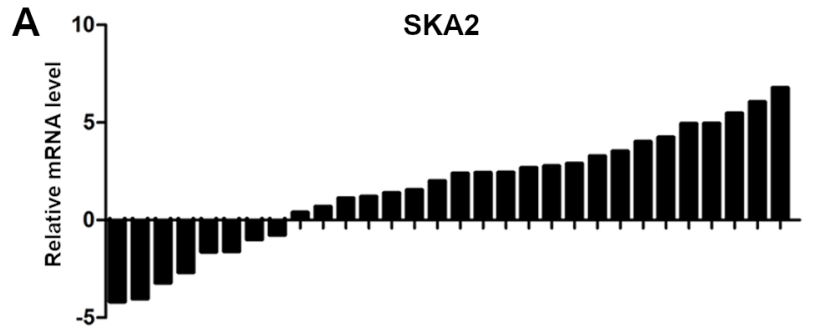

B
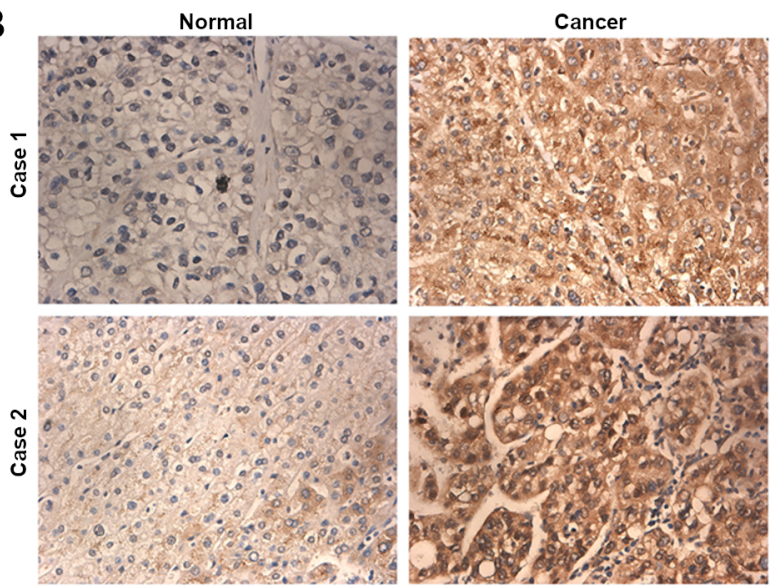

Hepatocellular Carcinoma with mRNA data (TCGA, $\mathrm{n}=360$ )

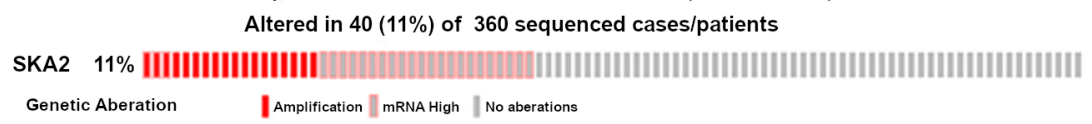

c

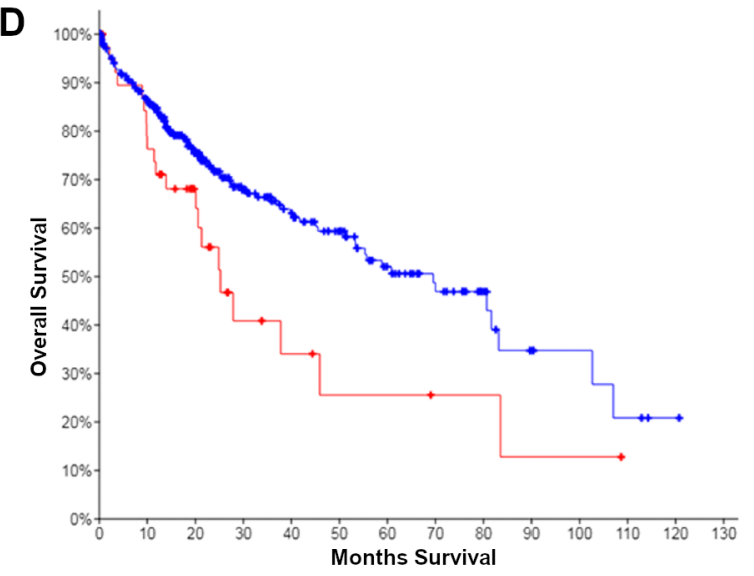

Logrank Test P-Value: 0.0161

- Cases with Alternation(s) in Query Gene(s)

- Cases without Alternation(s) in Query Gene(s)

Figure 1. SKA2 expression was upregulated in HCC tissues and correlated with HCC patient survival. A) SKA2 mRNA levels in 30 pairs of tumor samples (T) and matched normal hepatic tissues (N) determined by q-PCR. B) Immunohistochemistry staining of SKA2 in paired N and T tissues from two patients. C) SKA2 was altered in $11 \%$ of HCC patients (from TCGA database). D) SKA2 with alterations was correlated with worse overall survival in HCC patients (from TCGA database). 

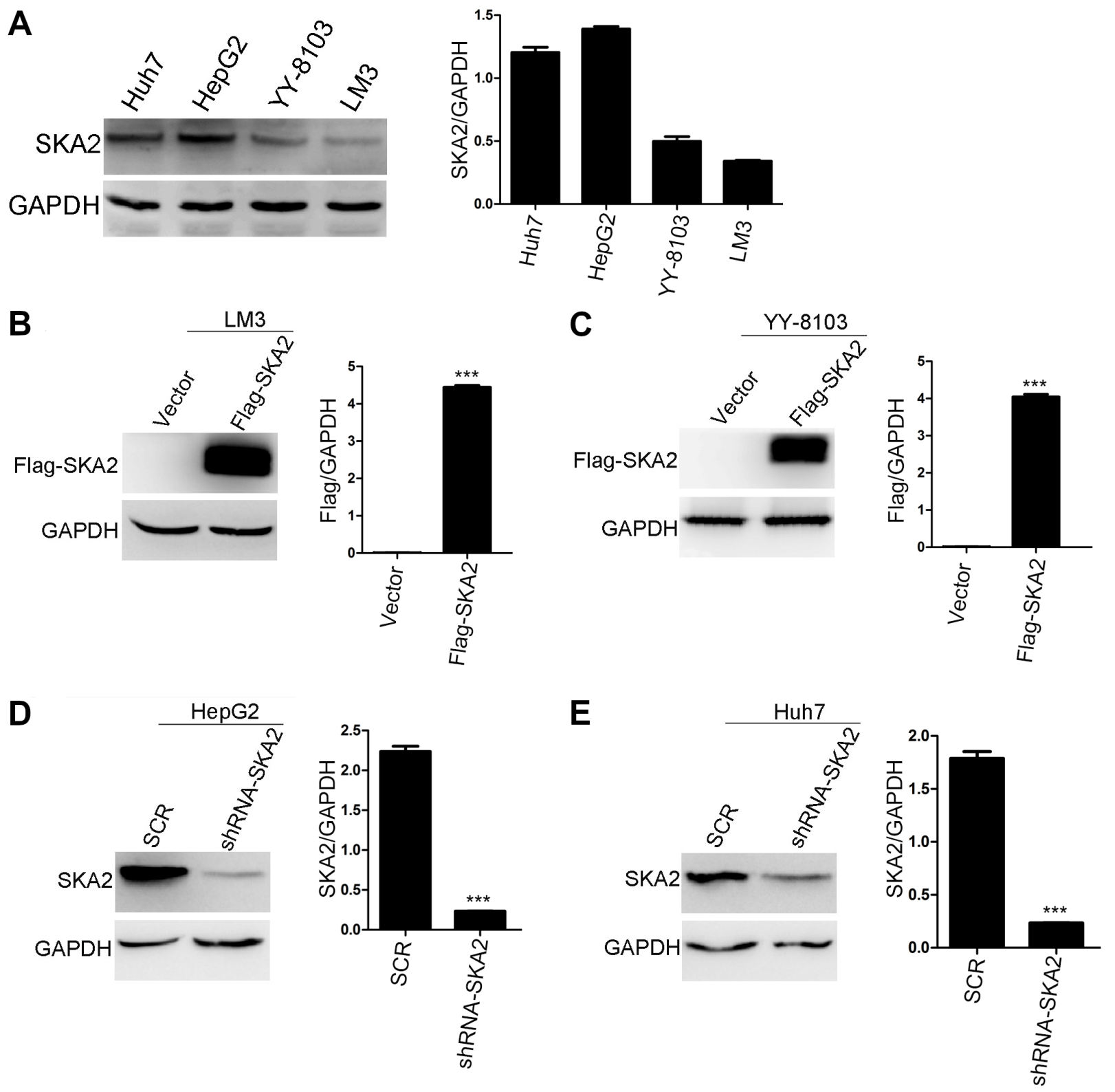

Figure 2. Overexpression and knockdown of SKA2 in HCC cell lines. A) Western blots showing SKA2 expression in four HCC cell lines: YY-8103, LM3, HepG2, and Huh7. Western blots showing the overexpression of SKA2 in B) LM3 cells and C) YY-8103 cells. Western blots showing the knocking down of SKA2 in D) HepG2 cells and E) Huh7 cells. Left panel: Result of western blot. Right panel: Grey analysis results of the western blot bands. GAPDH was used as a loading control.

showed that absorbance values of the LM3 and YY-8103 cells after transfection with SKA2 overexpression vectors were significantly higher than those of the untreated cells (all $\mathrm{p}<0.001$, Figure $3 \mathrm{~B}$ ). In addition, the transwell assays showed that the overexpression of SKA2 significantly promoted the invasion of LM3 and YY-8103 cells (Figure 3C).

Knocking down SKA2 expression inhibited the growth of HCC cell lines. Similar to the overexpression experiments, we tested the growth and invasion of the control and SKA2-shRNA cell lines. The MTT assays showed that the absorbance values of the HepG2 cells at 3, 4, 5 and 6 days after SKA2 downregulation were significantly lower than those of the untreated cells $(p<0.01$, Figure $4 A$ ). Similarly, the absorbance values of the Huh- 7 cells at 4, 5 and 6 days after SKA2 downregulation were also significantly lower than those of the untreated cells $(\mathrm{p}<0.01$, Figure $4 A)$. In addition, the crystal violet assays showed that absorbance values of the HepG2 and Huh-7 cells after SKA2 downregulation were significantly lower than those of the untreated cells (all $\mathrm{p}<0.001$, Figure 4B). Moreover, the transwell assays 
A

$$
\text { LM3 }
$$

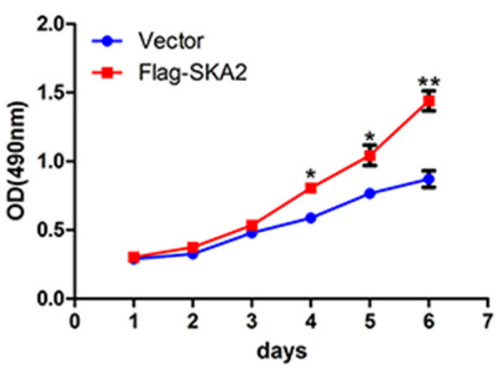

B

LM3
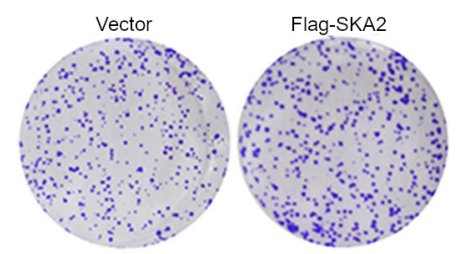

YY-8103
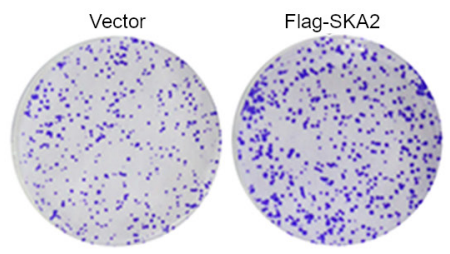

C

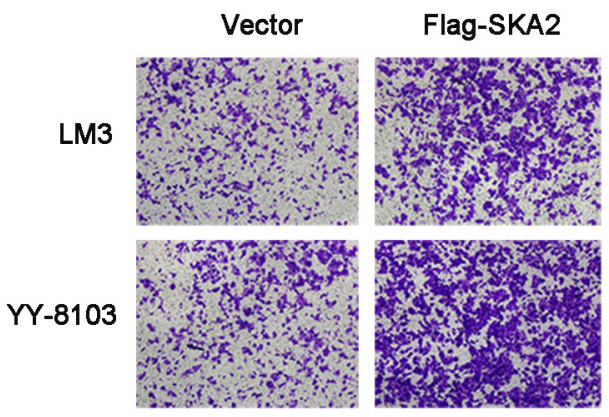

YY-8103
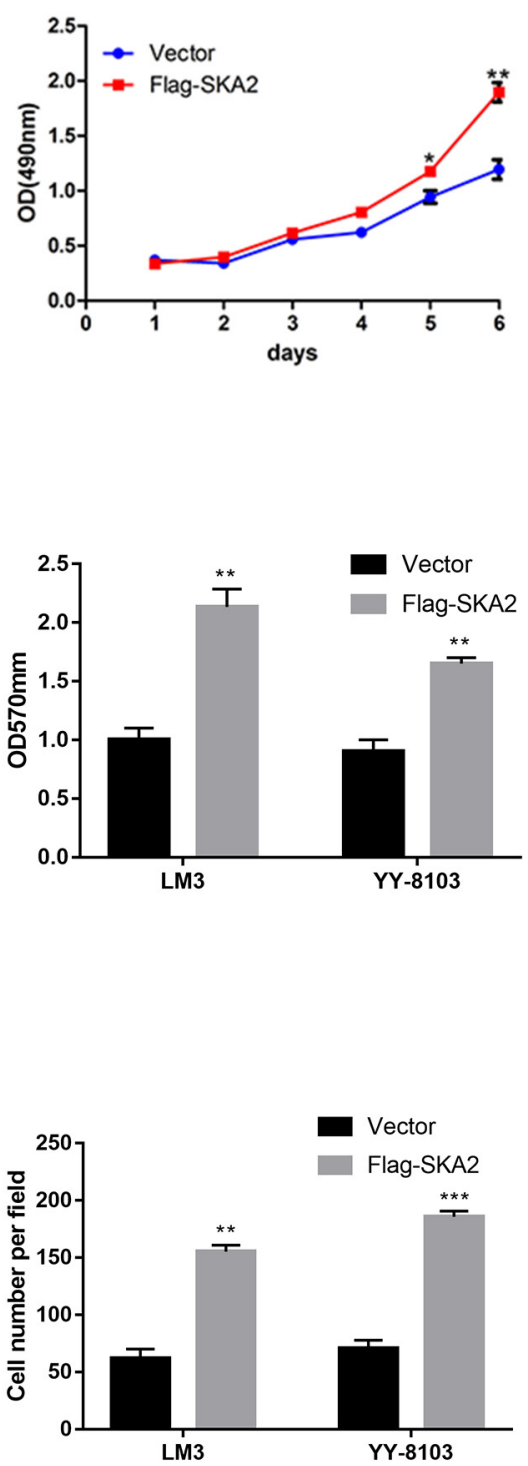

Figure 3. SKA2 overexpression promoted growth and invasion in HCC cells. A) The effects of SKA2 overexpression on the proliferation capacity of LM3 and YY-8103 cells were assessed by MTT assays. B) The effects of SKA2 overexpression on the proliferation capacity of LM3 and YY-8103 cells were assessed by crystal violet assays. C) The effects of SKA2 overexpression on the invasion of LM3 and YY-8103 cells were assessed by transwell assays. Quantitative blot analysis was shown on the right. Data shown are mean $\pm \operatorname{SD}$ from three independent experiments. ${ }^{\star} p<0.05,{ }^{* *} p<0.01,{ }^{* * *} p<0.001$, compared with the Vector group.

demonstrated that the knocking down of SKA2 significantly suppressed the invasion of HepG2 and Huh7 cells (Figure 3C).

SKA2 activated the $\beta$-catenin Signaling Pathway in HCC cells. It has been reported that Wnt $/ \beta$-catenin signaling pathway activation plays an important role in the development and progression of HCC. To explore the molecular mechanism of SKA2 on HCC, western blot analysis was performed to investigate the expression levels of total $\beta$-catenin protein levels in SKA2 overexpression and knock- down cell lines. As shown in Figure 5A-B, overexpression of SKA2 significantly increased the expression of total $\beta$-catenin in LM3 and YY-8103 cell lines. In turn, SKA2 downregulation significantly decreased the level of total $\beta$-catenin (Figures 5C-D). In addition, the expression level of Snail and Vimentin that were relevant to the tumor progression and metastasis were significantly increased by overexpression of SKA2 in LM3 and YY-8103 cells. However, the opposite effects were observed in HepG2 and Huh7 cells. Moreover, we also examined the expression level of some markers of 
A

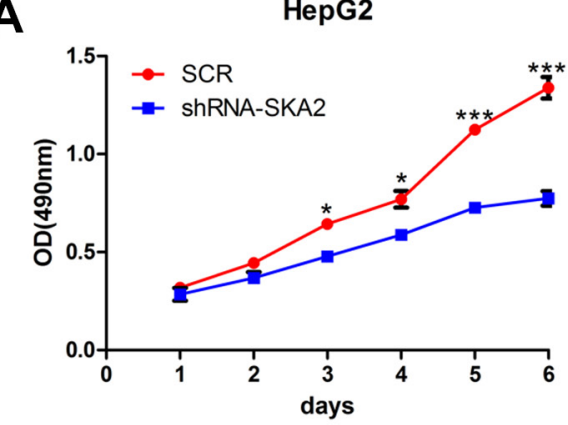

B

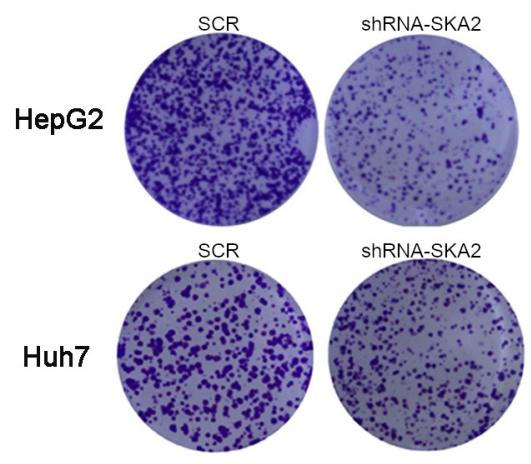

C

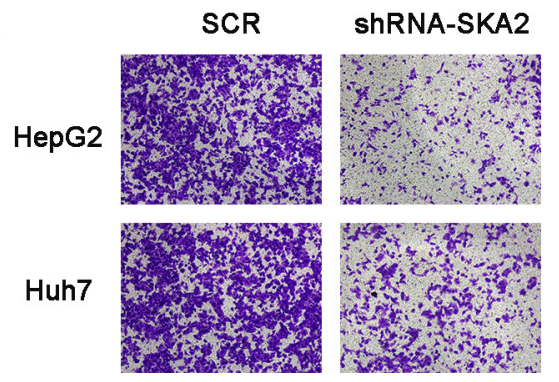

Huh7
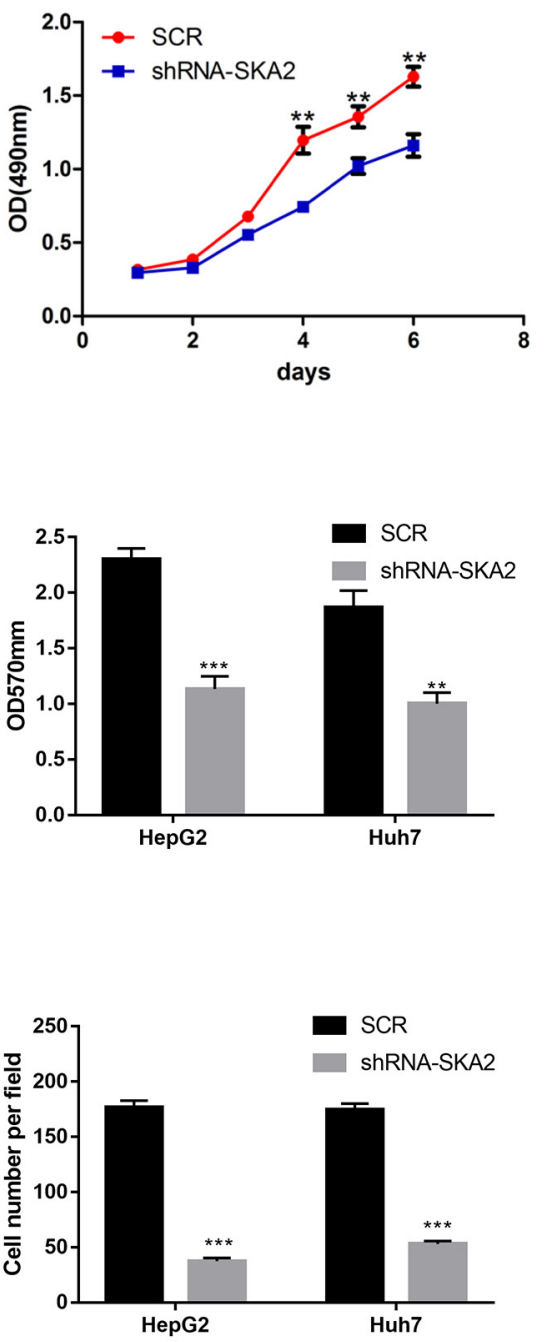

Figure 4. SKA2 knockdown inhibited growth in HCC cells. A) The effects of SKA2 knockdown on the proliferation capacity of HepG2 and Huh7 cells were assessed by MTT assays. B) The effects of SKA2 knockdown on the proliferation capacity of HepG2 and Huh7 cells were assessed by crystal violet assay. C) The effects of SKA2 knockdown on the invasion of HepG2 and Huh7 cells were assessed by transwell assays. Quantitative blot analysis was shown on the right. Data shown are mean \pm SD from three independent experiments. ${ }^{*} p<0.05,{ }^{* *} p<0.01,{ }^{* * *} p<0.001$, compared with the shRNA group.

other important signaling pathways, such as p-ERK in the RAF/MEK/ERK pathway, p-AKT, and p-mTOR in the PI3K/ AKT/mTOR pathway (Figure S2). We found that SKA2 did not influence these signaling pathways to promote the proliferation and invasion of HCC cells, which supported the finding that $\mathrm{Wnt} / \beta$-catenin pathway is exclusively involved in the upregulation of cell proliferation and invasion of hepatocellular carcinoma.

\section{Discussion}

The prognosis of patients with metastatic or recurrent HCC remains poor, with a 5 -year survival rate of $<15 \%$, despite some progress has been achieved in the diagnosis and treatment of HCC [21]. Therefore, exploring new regulating cancer genes and finding the new regulatory mechanism has become the key points in the field of HCC. Emerging evidence has shown that SKA2 was involved in tumorigenesis of various cancers, including breast cancer, lung cancer, and glioma [13-16].

In the current study, it is documented that the SKA2 mRNA levels and immunohistochemistry staining are significantly increased in HCC tissues, compared with normal tissues. Furthermore, the western analysis confirmed the successful overexpression and knockdown of SKA2 in HCC cell lines. Cell proliferation and invasion were significantly promoted following the overexpression of SKA2. In addition, the knocking down of SKA2 expression inhibited the growth 

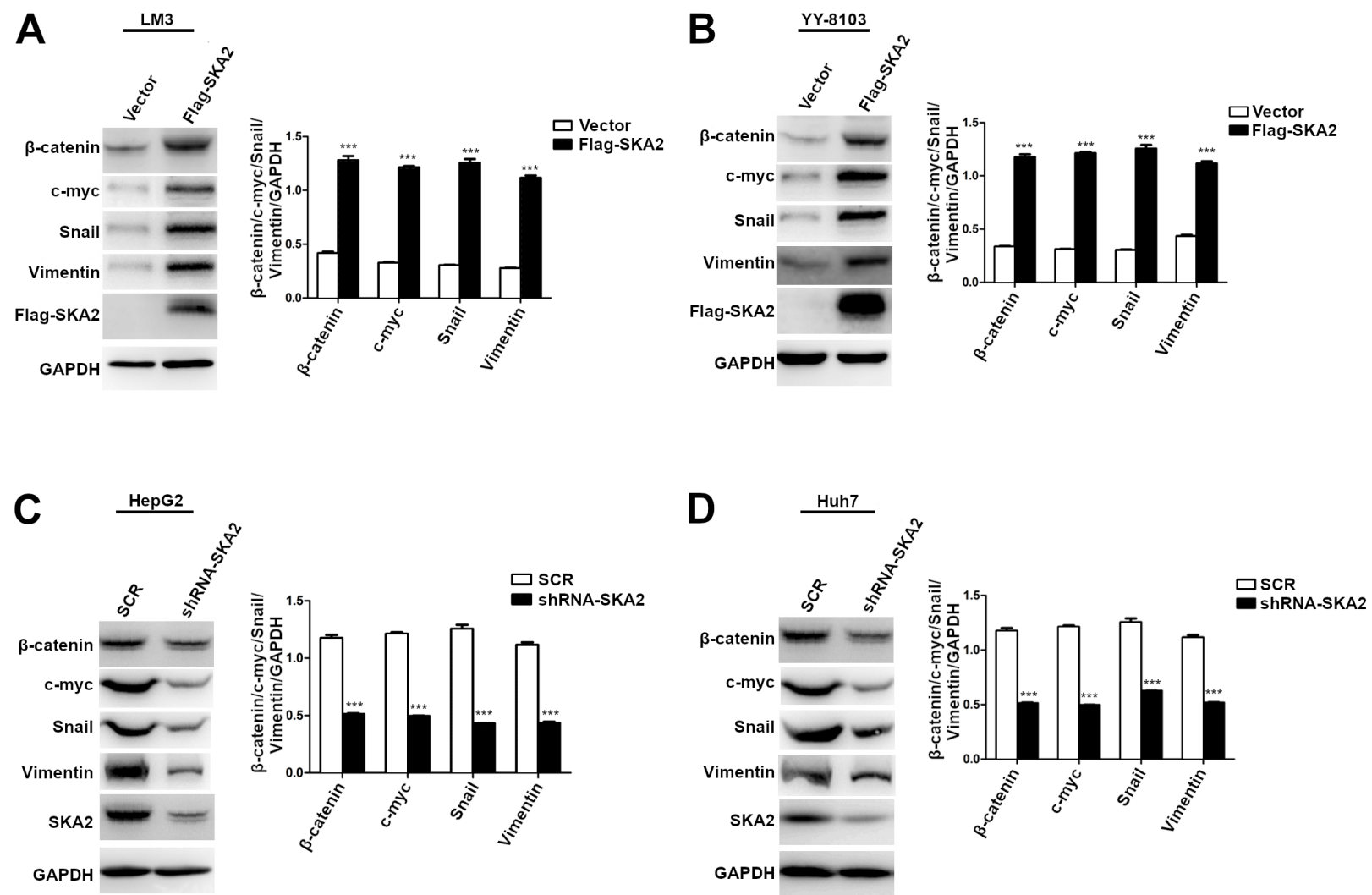

Figure 5. SKA2 activated the Wnt/ $\beta$-catenin signaling pathway. The overexpression SKA2 respectively increased the expression of total $\beta$-catenin, $\mathrm{c}$ myc, Snail, and Vimentin in LM3 (A) and YY-8103 cells (B) according to the western blot. Left panel: Result of western blot. Right panel: Grey analysis results of the western blot bands. GAPDH was used as a loading control. The knockdown SKA2 respectively decreased the expression of total $\beta$-catenin, c-myc, Snail, and Vimentin in HepG2 (C) and Huh7 (D) cells according to the western blot. Left panel: Result of western blot. Right panel: Grey analysis results of the western blot bands. GAPDH was used as a loading control.

and invasion of HCC cell lines. In experiments investigating the underlying mechanism, expression levels of total $\beta$-catenin were significantly increased by overexpression of SKA2. Meanwhile, the knockdown of SKA2 decreased the expression of total $\beta$-catenin that was relevant to the tumor progression. The present study revealed that SKA2 might promote proliferation and invasion of HCC cells by activating the $\mathrm{Wnt} / \beta$-catenin signaling pathway. It has been well reported that $\mathrm{Wnt} / \beta$-catenin signaling pathway was involved in cancer development and progression [22]. $\beta$-catenin is a core component of the canonical Wnt signaling pathway that regulates the transcription of several Wnt downstream target genes, such as c-myc, cyclinD1, and vimentin, leading to cell metastasis and invasion [23]. Moreover, our study found that the overexpression of SKA2 could increase the expression of Snail and Vimentin that were relevant to the tumor progression and metastasis. To the best of our knowledge, this is the first study revealing how SKA2 is involved in the proliferation and invasion of HCC.

In summary, our study demonstrated that SKA2 plays a critical role in the proliferation and invasion of HCC cells.
These results provide a better understanding of the mechanisms for the role of SKA2 in tumor development and might serve as a potential target in the diagnosis and/or treatment of HCC.

Supplementary information is available in the online version of the paper.

Acknowledgments: Regional science foundation of national natural science foundation of China (81460689).

\section{References}

1. LU XJ, SHI Y, CHEN JL, MA S. Kruppel-like factors in hepatocellular carcinoma. Tumour Biol 2015; 36: 533-541. https://doi.org/10.1007/s13277-015-3127-6

2. YU WB, RAO A, VU V, XU L, RAO JY et al. Management of centrally located hepatocellular carcinoma: Update 2016. World J Hepatol 2017; 9: 627-634. https://doi.org/10.4254/ wjh.v9.i13.627 
3. PORTOLANI N, CONIGLIO A, GHIDONI S, GIOVANELLI M, BENETTI A et al. Early and late recurrence after liver resection for hepatocellular carcinoma: prognostic and therapeutic implications. Ann Surg 2006; 243: 229-235. https:// doi.org/10.1097/01.sla.0000197706.21803.a1

4. HU J, GAO DZ. Distinction immune genes of hepatitisinduced hepatocellular carcinoma. Bioinformatics 2012; 28: 3191-3194. https://doi.org/10.1093/bioinformatics/bts624

5. MARQUARDT JU, GALLE PR, TEUFEL A. Molecular diagnosis and therapy of hepatocellular carcinoma (HCC): an emerging field for advanced technologies. J Hepatol 2012; 56: 267-275. https://doi.org/10.1016/j.jhep.2011.07.007

6. HANISCH A, SILLJE HH, NIGG EA. Timely anaphase onset requires a novel spindle and kinetochore complex comprising Ska1 and Ska2. EMBO J 2006; 25: 5504-5515. https:// doi.org/10.1038/sj.emboj.7601426

7. XIE M, BU Y. SKA2/FAM33A: A novel gene implicated in cell cycle, tumorigenesis, and psychiatric disorders. Genes Dis 2018; 6: 25-30. https://doi.org/10.1016/j.gendis.2018.11.001.

8. CHAN YW, JEYAPRAKASH AA, NIGG EA, SANTAMARIA A. Aurora B controls kinetochore-microtubule attachments by inhibiting Ska complex-KMN network interaction. J Cell Biol 2012; 196: 563-571. https://doi.org/10.1083/ jcb.201109001.

9. ZHANG Q, SIVAKUMAR S, CHEN Y, GAO H, YANG L et al. Ska3 Phosphorylated by Cdk1 Binds Ndc80 and Recruits Ska to Kinetochores to Promote Mitotic Progression. Curr Biol 2017; 27: 1477-1484. https://doi.org/10.1016/j. cub.2017.03.060

10. JEYAPRAKASH AA, SANTAMARIA A, JAYACHANDRAN U, CHAN YW, BENDA C et al. Structural and functional organization of the Ska complex, a key component of the kinetochore-microtubule interface. Mol Cell 2012; 46: 274-286. https://doi.org/10.1016/j.molcel.2012.03.005

11. RICE L, WATERS CE, ECCLES J, GARSIDE H, SOMMER $\mathrm{P}$ et al. Identification and functional analysis of SKA2 interaction with the glucocorticoid receptor. J Endocrinol 2008; 198: 499-509. https://doi.org/10.1677/JOE-08-0019

12. SHI W, GERSTER K, ALAJEZ NM, TSANG J, WALDRON L et al. MicroRNA-301 mediates proliferation and invasion in human breast cancer. Cancer Res 2011; 71: 2926-2937. https://doi.org/10.1158/0008-5472.CAN-10-3369

13. WANG Y, ZHANG C, MAI L, NIU Y, WANG Y et al. PRR11 and SKA2 gene pair is overexpressed and regulated by $\mathrm{p} 53$ in breast cancer. BMB Rep 2019; 52: 157-162.
14. REN Z, YANG T, ZHANG P, LIU K, LIU W et al. SKA2 mediates invasion and metastasis in human breast cancer via EMT. Mol Med Rep 2019; 19: 515-523. https://doi. org/10.3892/mmr.2018.9623

15. HE XJ, BIAN EB, MA CC, WANG C, WANG HL et al. Long non-coding RNA SPRY4-IT1 promotes the proliferation and invasion of U251 cells through upregulation of SKA2. Oncol Lett 2018; 15: 3977-3984. https://doi.org/10.3892/ ol.2018.7776

16. WANG Y, WENG H, ZHANG Y, LONG Y, LI Y et al. The PRR11-SKA2 Bidirectional Transcription Unit Is Negatively Regulated by p53 through NF-Y in Lung Cancer Cells. Int J Mol Sci 2017; 18: E534. https://doi.org/10.3390/ ijms18030534

17. GAO H, YIN FF, GUAN DX, FENG YX, ZHENG QW et al. Liver cancer: WISP3 suppresses hepatocellular carcinoma progression by negative regulation of beta-catenin/ TCF/LEF signalling. Cell Prolif 2019; 52: e12583. https://doi. org/10.1111/cpr.12583

18. WANG Y, LIU DP, CHEN PP, KOEFFLER HP, TONG XJ et al. Involvement of IFN regulatory factor (IRF)-1 and IRF-2 in the formation and progression of human esophageal cancers. Cancer Res 2007; 67: 2535-2543. https://doi. org/10.1158/0008-5472.CAN-06-3530

19. ZHANG C, ZHANG Y, LI Y, ZHU H, WANG Y et al. PRR11 regulates late-S to $\mathrm{G} 2 / \mathrm{M}$ phase progression and induces premature chromatin condensation (PCC). Biochem Biophys Res Commun 2015; 458: 501-508. https://doi.org/10.1016/j. bbrc.2015.01.139

20. WANG M, ZHANG Y, WANG T, ZHANG J, ZHOU Z et al. The USP7 Inhibitor P5091 Induces Cell Death in Ovarian Cancers with Different P53 Status. Cell Physiol Biochem 2017; 43: 1755-1766. https://doi.org/10.1159/000484062

21. European Association for the Study of the Liver. European Association for the Study of the Liver. EASL Clinical Practice Guidelines: Management of hepatocellular carcinoma. J Hepatol 2018; 69: 182-236. https://doi.org/10.1016/j. jhep.2018.03.019

22. YAO H, ASHIHARA E, MAEKAWA T. Targeting the Wnt/ beta-catenin signaling pathway in human cancers. Expert Opin Ther Targets 2011; 15: 873-887. https://doi.org/10.15 $17 / 14728222.2011 .577418$

23. MACDONALD BT, TAMAI K, HE X. Wnt/beta-catenin signaling: components, mechanisms, and diseases. Dev Cell 2009; 17: 9-26. https://doi.org/10.1016/j.devcel.2009.06.016 


\section{SKA2 promotes proliferation and invasion of hepatocellular carcinoma cells via activating the $\beta$-catenin signaling pathway}

D. WANG ${ }^{1}$, Y. J. SUO 2 , L. GONG 3 , S. Q. $\mathrm{LV}^{1, *}$

\section{Supplementary Information}

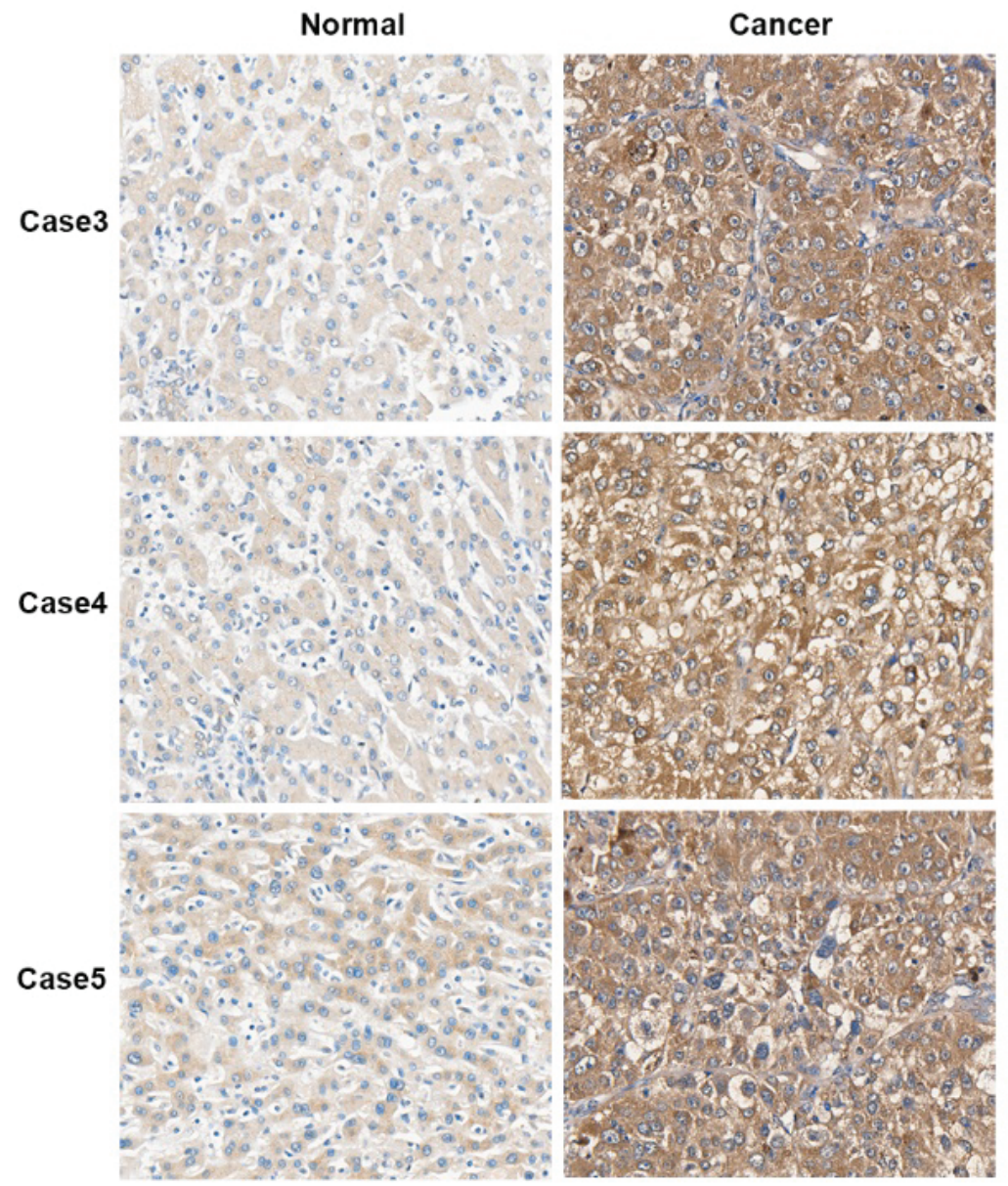

Figure S1. Immunohistochemistry staining of SKA2 in paired normal (N) and tumor (T) tissues from the other three patients. 
A

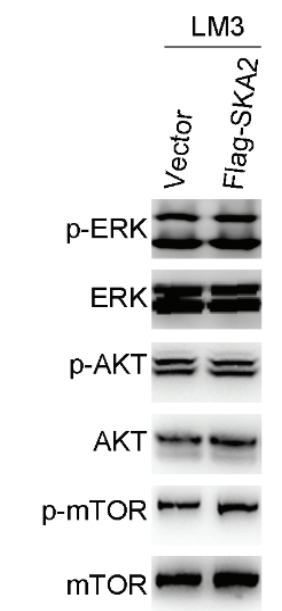

Flag-SKA2

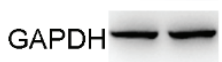

C

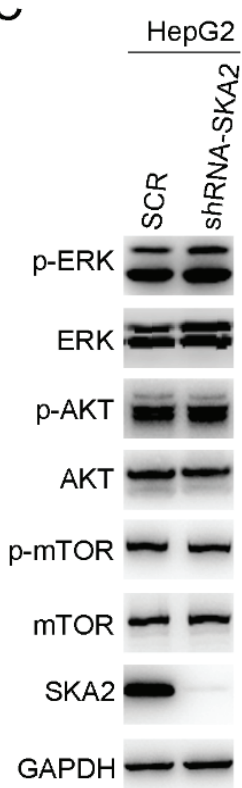

B

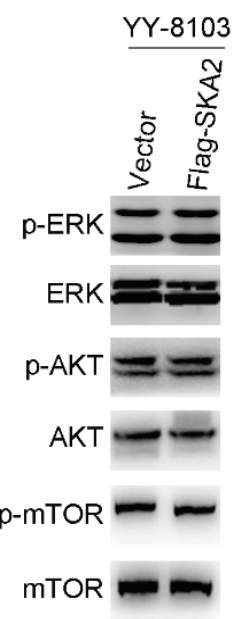

Flag-SKA2

GAPDH -

D

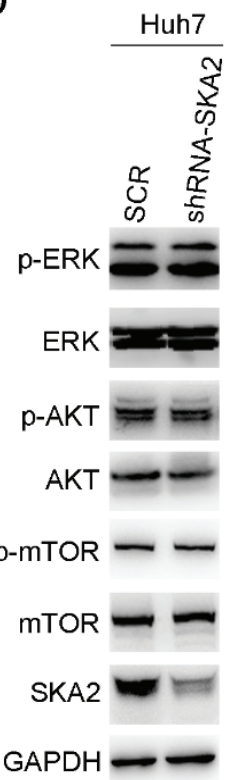

Figure S2. The expression level of p-ERK, ERK, p-AKT, AKT, p-mTOR, and mTOR among SKA2 overexpression and vector control groups were detected in LM3 (A) and YY-8103 cells (B) according to Western blot. GAPDH was used as a loading control. The expression level of p-ERK, ERK, p-AKT, AKT, p-mTOR, and mTOR among SKA2 knockdown and SCR control groups were detected in in HepG2 (C) and Huh7 (D) cells according to Western blot. GAPDH was used as a loading control. 